\title{
ON THE REPRESENTATION OF A FUNCTION BY CERTAIN FOURIER INTEGRALS*
}

\author{
BY \\ HARALD CRAMER
}

1. Introduction. Let us consider a complex-valued function $f(t)$ of the real variable $t$, which is bounded for all real $t$ and integrable in the Lebesgue sense over every finite interval. It is proposed to investigate the conditions under which $f(t)$ admits a representation of one of the following types:

$$
f(t)=\int_{-\infty}^{\infty} e^{i t x} d F(x),
$$

where $F(x)$ is real, bounded and never decreasing;

$$
f(t)=\int_{-\infty}^{\infty} e^{i t x} d G(x),
$$

where $G(x)$ is of bounded variation in $(-\infty, \infty)$; and

$$
f(t)=\int_{-\infty}^{\infty} e^{i t x} g(x) d x
$$

where $g(x)$ is absolutely integrable over $(-\infty, \infty)$. The functions $G(x)$ and $g(x)$ are not necessarily real.

We shall say that a representation of one of these types exists, whenever $f(t)$ is represented by the corresponding expression for almost all real $t$. If, in addition, we know a priori that $f(t)$ is continuous, it readily follows from elementary properties of the above integrals that our representation holds for all real $t$.

Now let us denote by $\mu(t)$ a function which satisfies the following conditions (1) and (2):

$$
\begin{gathered}
\int_{-\infty}^{\infty}|\mu(t)| d t \text { is finite, } \\
\mu(t)=\int_{-\infty}^{\infty} e^{i t x} m(x) d x,
\end{gathered}
$$

where $m(x)$ is real and never negative, and

$$
\mu(0)=\int_{-\infty}^{\infty} m(x) d x=1 .
$$

* Presented to the Society, February 25, 1939; received by the editors January 31, 1939. 
The functions $\mu(t)=e^{-t^{2} / 2}, \mu(t)=e^{-|t|}$, and

$$
\mu(t)= \begin{cases}1-|t|, & |t| \leqq 1, \\ 0, & |t| \geqq 1,\end{cases}
$$

are examples of functions satisfying these conditions. The corresponding $m(x)$-functions are, respectively,

$$
\frac{1}{(2 \pi)^{1 / 2}} e^{-x^{2} / 2}, \quad \frac{1}{\pi\left(1+x^{2}\right)}, \quad \frac{1-\cos x}{\pi x^{2}} .
$$

For any positive $\epsilon$ we denote by $g_{\epsilon}(x)$ the function defined for all real $x$ by the absolutely convergent integral

$$
g_{\epsilon}(x)=\frac{1}{2 \pi} \int_{-\infty}^{\infty} e^{-i t x} \mu(\epsilon t) f(t) d t .
$$

Obviously $g_{\epsilon}(x)$ is bounded and everywhere continuous.

We then have for any particular $\mu(t)$ satisfying (1) and (2) the following necessary and sufficient conditions for the existence of a representation of $f(t)$ according to $(\mathrm{F}),(\mathrm{G})$, or $(\mathrm{g})$ :

Type $(\mathrm{F}) . g_{\epsilon}(x)$ should be real and never negative for $0<\epsilon<1$ and for all real $x$.

Type (G). $\int_{-\infty}^{\infty}\left|g_{\epsilon}(x)\right| d x<$ const. for $0<\epsilon<1$.

Type $(\mathrm{g}) . g_{\epsilon}(x)$ should satisfy the condition for type $(\mathrm{G})$, and further

$$
\lim _{\substack{\epsilon \rightarrow 0 \\ c \rightarrow 0}} \int_{-\infty}^{\infty}\left|g_{\epsilon}(x)-g_{\epsilon^{\prime}}(x)\right| d x=0 .
$$

If a given function $f(t)$ satisfies one of these conditions for one particular function $\mu(t)$, it follows that the same condition is automatically satisfied for all $\mu(t)$ satisfying (1) and (2).

Proofs of the conditions will be given in $\$ \S 3-5$. In $\$ 7$, it will be shown that similar conditions hold for functions $f\left(t_{1}, \cdots, t_{k}\right)$ of any number of variables.

2. A particular case. Choosing for $\mu(t)$ the particular function given by (3), we obtain, writing $A=1 / \epsilon$,

$$
\begin{aligned}
g_{\epsilon}(x) & =\frac{1}{2 \pi} \int_{-A}^{A}\left(1-\frac{|t|}{A}\right) f(t) e^{-i t x} d t \\
& =\frac{1}{2 \pi A} \int_{0}^{A} \int_{0}^{A} f(t-u) e^{-i x(t-u)} d t d u .
\end{aligned}
$$

In this particular case, our conditions are analogous to those given by 
Hausdorff [4] with respect to the problem of representing a sequence of numbers $c_{k},(k=0, \pm 1, \pm 2, \cdots)$, in the form

$$
c_{k}=\int_{0}^{2 \pi} e^{i k x} d F(x)
$$

or in one of the similar forms corresponding to $(G)$ or $(g)$.

Our condition for type (F) constitutes, in the particular case when $\mu(t)$ is given by (3), a simplified form of a well known theorem due to Bochner (cf. \$7). For type (G), Bochner [2] and Schoenberg [6] have given a necessary and sufficient condition which is, however, fundamentally different from ours.

Some applications of our conditions to the theory of random processes will be given in a forthcoming paper.

3. Representation of type $(F)$. In the case of a representation

$$
f(t)=\int_{-\infty}^{\infty} e^{i t x} d F(x)
$$

with a real, bounded, and never decreasing $F(x)$, it is almost obvious that our condition is necessary. We obtain, in fact, from (4)

$$
\begin{aligned}
g_{\epsilon}(x) & =\frac{1}{2 \pi} \int_{-\infty}^{\infty} e^{-i t x} \mu(\epsilon t) d t \int_{-\infty}^{\infty} e^{i t y} d F(y) \\
& =\frac{1}{2 \pi} \int_{-\infty}^{\infty} d F(y) \int_{-\infty}^{\infty} e^{-i t(x-y)} \mu(\epsilon t) d t,
\end{aligned}
$$

the inversion of the order of integration being justified by the absolute convergence of the integrals. According to (1) and (2) we have, however, almost everywhere

$$
m(x)=\frac{1}{2 \pi} \int_{-\infty}^{\infty} e^{-i t x} \mu(t) d t,
$$

so that $g_{\epsilon}(x)$ is given by the "Faltung"

$$
g_{\epsilon}(x)=\frac{1}{\epsilon} \int_{-\infty}^{\infty} m\left(\frac{x-y}{\epsilon}\right) d F(y),
$$

which is obviously real and never negative.

In order to show that the condition is also sufficient, we consider the identity

$$
\int_{-2 A}^{2 A}\left(1-\frac{|x|}{2 A}\right) e^{-i t x} d x=2 A\left(\frac{\sin A t}{A t}\right)^{2}
$$


which holds for every $A>0$. Multiplying by $(2 \pi)^{-1} \mu(\epsilon t) f(t) d t$ and integrating with respect to $t$ over $(-\infty, \infty)$, we obtain, according to (4),

$$
\int_{-2 A}^{2 A}\left(1-\frac{|x|}{2 A}\right) g_{\epsilon}(x) d x=\frac{1}{\pi} \int_{-\infty}^{\infty}\left(\frac{\sin t}{t}\right)^{2} \mu(\epsilon t / A) f(t / A) d t .
$$

Now $|\mu(t)| \leqq 1$ by (2), and $f(t)$ is bounded by hypothesis; say $|f(t)| \leqq c$. Thus if $g_{\epsilon}(x)$ is real and never negative, we conclude

$$
\int_{-2 A}^{2 A}\left(1-\frac{|x|}{2 A}\right) g_{\epsilon}(x) d x \leqq c
$$

for $0<\epsilon<1$ and for all positive $A$. This obviously implies

$$
\int_{-\infty}^{\infty} g_{\epsilon}(x) d x \leqq c
$$

for $0<\epsilon<1$.

From (4) and (6) we then obtain for almost all values of $x$

$$
\mu(\epsilon t) f(t)=\int_{-\infty}^{\infty} e^{i t x} g_{\epsilon}(x) d x .
$$

Now, since both $\mu(t)$ and the integral are continuous functions of $t$, it follows that it is possible to find a continuous function $f^{*}(t)$ which coincides with $f(t)$ for almost all real $t$. We then have

$$
\mu(\epsilon t) f^{*}(t)=\int_{-\infty}^{\infty} e^{i t x} g_{\epsilon}(x) d x
$$

for all real $t$ and for $0<\epsilon<1$.

Consider now the last relation for a sequence of values of $\epsilon$ tending to zero. As $\mu(0)=1$, the left-hand side tends to $f^{*}(t)$ uniformly in every finite interval. According to a fundamental theorem on characteristic functions due to Lévy [5] (cf. also Bochner [1]), we then have for all real $t$

$$
f^{*}(t)=\int_{-\infty}^{\infty} e^{i t x} d F(x)
$$

where $F(x)$ is real and never decreasing. As $f^{*}(t)=f(t)$ for almost all $t$, this proves our assertion.

4. Representation of type $(\mathrm{G})$. $\dagger$ If we have for almost all $t$

$$
f(t)=\int_{-\infty}^{\infty} e^{i t x} d G(x),
$$

$\dagger$ The author is indebted to Mr. E. Frithiofson of Lund for a remark leading to a simplification of the condition for this type. 
where $G(x)$ is of bounded variation in $(-\infty, \infty)$, we obtain as in the preceding paragraph

$$
g_{\epsilon}(x)=\frac{1}{\epsilon} \int_{-\infty}^{\infty} m\left(\frac{x-y}{\epsilon}\right) d G(y)
$$

and thus, $m(x)$ being never negative,

$$
\int_{x_{1}}^{x_{2}}\left|g_{\epsilon}(x)\right| d x \leqq \int_{-\infty}^{\infty}|d G(y)| \int_{\left(x_{1}-y\right) / \epsilon}^{\left(x_{2}-y\right) / \epsilon} m(x) d x .
$$

Hence we obtain, using (2b),

$$
\int_{-\infty}^{\infty}\left|g_{\epsilon}(x)\right| d x \leqq \int_{-\infty}^{\infty}|d G(y)|
$$

for $0<\epsilon<1$. Thus our condition is necessary.

In order to show that the condition is also sufficient we observe that, owing to the convergence of $\int_{-\infty}^{\infty}\left|g_{\epsilon}(x)\right| d x$, the relation (4) may be converted into (7) for almost all real $t$. As in the preceding section, it follows that there is a continuous function $f^{*}(t)$ which coincides with $f(t)$ for almost all real $t$. We then have as before

$$
\mu(\epsilon t) f^{*}(t)=\int_{-\infty}^{\infty} e^{i t x} g_{\epsilon}(x) d x
$$

for all real $t$ and for $0<\epsilon<1$. Putting

$$
G_{\epsilon}(x)=\int_{-\infty}^{x} g_{\epsilon}(y) d y,
$$

we may write this as

$$
\mu(\epsilon t) f^{*}(t)=\int_{-\infty}^{\infty} e^{i t x} d G_{\epsilon}(x) .
$$

When $\epsilon$ tends to zero, the left-hand side of this relation tends to $f^{*}(t)$ uniformly in every finite interval. On the other hand, $\int_{-\infty}^{\infty}\left|g_{\epsilon}(x)\right| d x$ is uniformly bounded for $0<\epsilon<1$, so that $G_{\epsilon}(x)$ is of uniformly bounded variation in $(-\infty, \infty)$. It is well known that we can always find a sequence $\epsilon_{1}, \epsilon_{2}, \cdots$ tending to zero and a function $G(x)$ of bounded variation in $(-\infty, \infty)$ such that

$$
G(x)=\lim _{n \rightarrow \infty} G_{\epsilon_{n}}(x)=\lim _{n \rightarrow \infty} \int_{-\infty}^{x} g_{\epsilon_{n}}(y) d y
$$

in every point of continuity $x$ of $G(x)$. It then follows from a lemma given by 
Bochner [2, p. 274], that we have for all real $t$

$$
f^{*}(t)=\int_{-\infty}^{\infty} e^{i \ell x} d G(x)
$$

As $f^{*}(t)=f(t)$ for almost all $t$, this proves our assertion.

For a later purpose it will now be shown that, if our condition for type $(G)$ is satisfied, then the integral

$$
\int_{-\infty}^{\infty}\left|g_{\epsilon}(x)\right| d x
$$

is uniformly convergent for $0<\epsilon<1$. If the condition is satisfied, we already know that $f(t)$ admits a representation of type (G). Now let $\delta>0$ be given. We can then choose $y_{0}>0$ and $x_{0}>y_{0}$ such that

$$
\int_{y_{0}}^{\infty}|d G(y)|<\delta, \quad \int_{x_{0}-y_{0}}^{\infty} m(x) d x<\delta .
$$

Obviously $x_{0}$ and $y_{0}$ can be chosen independently of $\epsilon$. For $x_{2}>x_{1}>x_{0}$ and for $0<\epsilon<1$, we then conclude from (8) and (2b) that

$$
\begin{aligned}
\int_{x_{1}}^{x_{2}}\left|g_{\epsilon}(x)\right| d x & <\delta \int_{-\infty}^{y_{0}}|d G(y)|+\int_{y_{0}}^{\infty}|d G(y)| \\
& <\delta\left[1+\int_{-\infty}^{\infty}|d G(y)|\right] .
\end{aligned}
$$

A similar inequality evidently holds for negative values of $x_{1}$ and $x_{2}$, and thus the uniform convergence of (10) is established.

5. Representation of type (g). As in the preceding cases, we begin by proving that our condition is necessary. Any representation of type (g) being a particular case of type $(\mathrm{G})$, it is obvious that the first part of the condition is necessary. It thus remains to show that, if

$$
f(t)=\int_{-\infty}^{\infty} e^{i t x} g(x) d x
$$

holds for almost all $t$, where $g(t)$ is absolutely integrable over $(-\infty, \infty)$, then

$$
\lim _{\substack{c \rightarrow 0 \\ \boldsymbol{c}^{\prime} \rightarrow 0}} \int_{-\infty}^{\infty}\left|g_{\epsilon}(x)-g_{\epsilon^{\prime}}(x)\right| d x=0 .
$$

As $\left|g_{\epsilon}-g_{\epsilon^{\prime}}\right| \leqq\left|g-g_{\epsilon}\right|+\left|g-g_{\epsilon^{\prime}}\right|$, it is only necessary to prove that

$$
\lim _{\epsilon \rightarrow 0} \int_{-\infty}^{\infty}\left|g(x)-g_{\epsilon}(x)\right| d x=0 \text {. }
$$


According to the preceding section, it follows from the first part of the condition that the integral (10) converges uniformly for $0<\epsilon<1$. Given $\delta>0$, we can thus choose $x_{0}=x_{0}(\delta)$ such that

$$
\int_{|x|>x_{0}}\left|g(x)-g_{\epsilon}(x)\right| d x<\delta
$$

for $0<\epsilon<1$.

We now choose a function $g^{*}(x)$, bounded and continuous for all real $x$, such that

$$
\int_{-\infty}^{\infty}\left|g(x)-g^{*}(x)\right| d x<\delta
$$

with

$$
\left|g^{*}(x)\right|<K=K(\delta)
$$

and we put

$$
g_{\epsilon}^{*}(x)=\frac{1}{\epsilon} \int_{-\infty}^{\infty} m\left(\frac{x-y}{\epsilon}\right) g^{*}(y) d y .
$$

We then have

$$
\begin{aligned}
\int_{-x_{0}}^{x_{0}}\left|g(x)-g_{\epsilon}(x)\right| d x \leqq & \int_{-x_{0}}^{x_{0}}\left|g(x)-g^{*}(x)\right| d x+\int_{-x_{0}}^{x_{0}}\left|g^{*}(x)-g_{\epsilon}^{*}(x)\right| d x \\
& +\int_{-x_{0}}^{x_{0}}\left|g_{\epsilon}^{*}(x)-g_{\epsilon}(x)\right| d x .
\end{aligned}
$$

According to (13), the first term on the right-hand side is less than $\delta$. We further have, using $(2 b)$,

$$
g^{*}(x)-g_{\epsilon}^{*}(x)=\frac{1}{\epsilon} \int_{-\infty}^{\infty} m\left(\frac{x-y}{\epsilon}\right)\left(g^{*}(x)-g^{*}(y)\right) d y .
$$

Now, $g^{*}(x)$ is uniformly continuous in every finite interval. The numbers $\delta$ and $x_{0}$ being given, we can thus choose $h=h\left(\delta, x_{0}\right)$ such that for $|x|<x_{0},|x-y|<h$ we have

$$
\left|g^{*}(x)-g^{*}(y)\right|<\delta / x_{0} .
$$

We can further choose $y_{0}=y_{0}\left(\delta, x_{0}, K\right)$ such that

$$
\int_{|y|>\nu_{0}} m(y) d y<\frac{\delta}{2 K x_{0}} .
$$

For any $\epsilon$ such that $0<\epsilon<h / y_{0}$, we then obtain from (15) 
and

$$
\left|g^{*}(x)-g_{\epsilon}^{*}(x)\right|<\frac{\delta}{x_{0}}+2 K \int_{|y|>h / \epsilon} m(y) d y<\frac{2 \delta}{x_{0}}
$$

$$
\int_{-x_{0}}^{x_{0}}\left|g^{*}(x)-g_{\epsilon}^{*}(x)\right| d x<4 \delta
$$

Finally, we have

$$
g_{\epsilon}^{*}(x)-g_{\epsilon}(x)=\frac{1}{\epsilon} \int_{-\infty}^{\infty} m\left(\frac{x-y}{\epsilon}\right)\left(g^{*}(y)-g(y)\right) d y,
$$

and hence by (13)

$$
\begin{aligned}
\int_{-x_{0}}^{x_{0}}\left|g_{\epsilon}^{*}(x)-g_{\epsilon}(x)\right| d x & \leqq \int_{-\infty}^{\infty}\left|g^{*}(y)-g(y)\right| d y \int_{\left(-x_{0}-y\right) / e}^{\left(x_{0}-y\right) / e} m(x) d x \\
& \leqq \int_{-\infty}^{\infty}\left|g^{*}(y)-g(y)\right| d y<\delta .
\end{aligned}
$$

From (12), (14), (16), and (17) we then obtain

$$
\int_{-\infty}^{\infty}\left|g(x)-g_{\epsilon}(x)\right| d x<7 \delta
$$

for all sufficiently small $\epsilon>0$, so that (11) is proved.

We now have to show that our condition is sufficient. From the first part of the condition, it follows by the preceding paragraph that we have for almost all real $t$

$$
f(t)=\int_{-\infty}^{\infty} e^{i t x} d G(x)
$$

where $G(x)$ is of bounded variation in $(-\infty, \infty)$, and according to (9)

$$
G(x)=\lim _{n \rightarrow \infty} G_{\epsilon_{n}}(x)=\lim _{n \rightarrow \infty} \int_{-\infty}^{x} g_{\epsilon_{n}}(y) d y
$$

in every point of continuity $x$ of $G(x)$.

From the second part of the condition it follows, however, that there is a function $g(x)$, absolutely integrable over $(-\infty, \infty)$, such that

$$
\lim _{n \rightarrow \infty} \int_{-\infty}^{\infty}\left|g(y)-g_{\epsilon_{n}}(y)\right| d y=0 .
$$

Hence we obtain for all real $x$ 


$$
\lim _{n \rightarrow \infty} G_{\epsilon_{n}}(x)=\lim _{n \rightarrow \infty} \int_{-\infty}^{x} g_{\epsilon_{n}}(y) d y=\int_{-\infty}^{x} g(y) d y .
$$

It then finally follows from (18) that

for almost all $x$, and

$$
G(x)=\int_{-\infty}^{x} g(y) d y
$$

$$
f(t)=\int_{-\infty}^{\infty} e^{i t x} g(x) d x
$$

for almost all $t$, so that the proof is completed.

If the first part of our condition for type ( $\mathrm{g}$ ) is replaced by the condition given above for type $(F)$, it is readily seen that we obtain a necessary and sufficient condition for representation of type (g) with a real and non-negative $g(x)$.

It may be worth while to point out that the first part of our condition for type ( $\mathrm{g}$ ) is not contained in the second part. This is shown by the example

$$
f(t)=\left\{\begin{array}{l}
i(-1-t),-1<t<0, \\
i(1-t), 0<t<1, \\
0, t=0,|t| \geqq 1
\end{array}\right.
$$

In the particular case when $\mu(t)$ is given by (3), this function yields for $0<\epsilon<1$

$$
g_{\epsilon}(x)=\frac{x-\sin x}{\pi x^{2}}+\epsilon \frac{x \sin x-2(1-\cos x)}{\pi x^{3}},
$$

so that the second part of the condition is satisfied but not the first part. Accordingly, no representation of any of our three types exists, which is also directly seen from the behaviour of $f(t)$ near $t=0$.

6. The case of an unbounded $f(t)$. In all the preceding paragraphs it has been a priori assumed that $f(t)$ is bounded. It will, however, be seen that this assumption has only been used on two occasions; namely (a) to ensure the absolute convergence of the integral (4) which defines $g_{\epsilon}(x)$, and (b) for the proof that our condition for type $(\mathrm{F})$ is sufficient.

Let us now omit this assumption and consider the class of all functions $f(t)$ which are integrable over any finite interval. Let us further choose for $\mu(t)$ the particular function given by (3). As this function is equal to zero for $|t| \geqq 1$, it is obvious that the integral (4) will still be absolutely convergent for any positive $\epsilon$.

Thus the conditions for types $(\mathrm{G})$ and $(\mathrm{g})$ remain true under the present con- 
ditions, while in the condition for type $(\mathrm{F})$ it will have to be explicitly stated that $|f(t)|$ should be less than a constant $K$ for almost all values of $t$.

The necessity of this addition to the condition for type $(F)$ is shown by the example $f(t)=|t|^{-\alpha},(0<\alpha<1)$, where obviously no $K$ can be found such that $|f(t)|<K$ for almost all $t$. The corresponding function $g_{\epsilon}(x)$ can be shown to be positive for $0<\epsilon<1$ and for all real $x$, although evidently no representation of type $(\mathrm{F})$ exists.

7. Functions of several variables. So far we have only considered functions $f(t)$ of a single variable $t$. All our considerations can, however, be extended to functions $f\left(t_{1}, \cdots, t_{k}\right)$ of any finite number of real variables. This requires only a straightforward generalization of our above arguments, based on the elementary properties of Fourier integrals in several variables. The only delicate point arising in this connection is the generalization to several variables of Bochner's lemma used in the proof of our condition for type $(G)$. This generalization is, however, easily performed by means of a general induction method due to Cramér and Wold (Cramér [3, p. 104]).

We obtain in this way direct generalizations of our above conditions, the auxiliary functions $\mu(t)$ and $g_{\epsilon}(x)$ being replaced by the functions of $k$ variables obtained when, in (1), (2), and (4), we regard $x, t$, and $\epsilon t$ as abbreviations for $\left(x_{1}, \cdots, x_{k}\right),\left(t_{1}, \cdots, t_{k}\right)$, and $\left(\epsilon t_{1}, \cdots, \epsilon t_{k}\right)$, respectively, and put $t x=t_{1} x_{1}+\cdots+t_{k} x_{k}$, the integrals being taken over the $k$-dimensional euclidean space $R_{k}$. Moreover, in the definition (4) of $g_{\epsilon}(x)$ the factor $1 / 2 \pi$ has to be replaced by $1 /(2 \pi)^{k}$.

For $\mu\left(t_{1}, \cdots, t_{k}\right)$ we may, for example, choose any function of the form $\mu\left(t_{1}\right) \mu\left(t_{2}\right) \cdots \mu\left(t_{k}\right)$, where $\mu(t)$ satisfies the conditions (1) and (2). The definition (4) of $g_{\epsilon}(x)$ will then be replaced by

$$
\begin{aligned}
& g_{\epsilon}\left(x_{1}, \cdots, x_{k}\right) \\
& \quad=\frac{1}{(2 \pi)^{k}} \int_{R_{k}} e^{-i\left(t_{1} x_{1}+\cdots+t_{k} x_{k}\right)} \mu\left(\epsilon t_{1}\right) \cdots \mu\left(\epsilon t_{k}\right) f\left(t_{1}, \cdots, t_{k}\right) d t_{1} \cdots d t_{k} .
\end{aligned}
$$

In particular, we obtain in this way the following new characterization of the class of positive definite functions of $k$ variables as defined by Bochner $[1$, p. 406]. Bochner has established the identity of this class with the class of functions represented for all real $t_{r}$ by the expression

$$
f\left(t_{1}, \cdots, t_{k}\right)=\int_{R_{k}} e^{i\left(t_{1} x_{1}+\cdots+t_{k} x_{k}\right)} d F\left(x_{1}, \cdots, x_{k}\right),
$$

where $F$ is real, bounded, and, for each particular $x_{r}$, never decreasing. The class of positive definite functions such that $f(0, \cdots, 0)=1$ is thus identical 
with the class of characteristic functions of $k$-dimensional random variables in the sense of the theory of probability (cf. Cramér [3]). Using our generalized condition for type $(F)$ we then conclude:

For any particular $\mu(t)$ satisfying (1) and (2) a necessary and sufficient condition that a given bounded and continuous function $f\left(t_{1}, \cdots, t_{k}\right)$ should be positive definite is that $g_{\epsilon}\left(x_{1}, \cdots, x_{k}\right)$ as defined by (19) should be real and never negative for $0<\epsilon<1$ and for all real $x_{1}, \cdots, x_{k}$.

Choosing, in particular, in (19) the special function $\mu(t)$ given by (3), we obtain in analogy with (5), writing $A=1 / \epsilon$,

$$
\begin{aligned}
g_{\epsilon}\left(x_{1}, \cdots, x_{k}\right)= & \frac{1}{(2 \pi)^{k}} \int_{-A}^{A} \cdots \int_{-A}^{A} f\left(t_{1}, \cdots, t_{k}\right) \\
& \cdot \prod_{r=1}^{k}\left[\left(1-\frac{\left|t_{r}\right|}{A}\right) e^{-i t_{r} x_{r}}\right] d t_{1} \cdots d t_{r} \\
= & \frac{1}{(2 \pi A)^{k}} \int_{0}^{A} \cdots \int_{0}^{A} f\left(t_{1}-u_{1}, \cdots, t_{k}-u_{k}\right) \\
& \cdot \exp \left(-i \sum_{1}^{k} x_{r} t_{r}\right) \cdot \exp \left(i \sum_{1}^{k} x_{r} u_{r}\right) d t_{1} \cdots d t_{k} d u_{1} \cdots d u_{k} .
\end{aligned}
$$

Now Bochner's original condition for a positive definite function requires that

$$
\begin{array}{r}
\int_{a}^{\Lambda} \cdots \int_{a}^{\Lambda} f\left(t_{1}-u_{1}, \cdots, t_{k}-u_{k}\right) \rho\left(t_{1}, \cdots, t_{k}\right) \\
\qquad \overline{\frac{\rho\left(u_{1}, \cdots, u_{k}\right)}{} d t_{1} \cdots d t_{k} d u_{1} \cdots d u_{k} \geqq 0}
\end{array}
$$

for all real $a, A$ and for all continuous functions $\rho\left(t_{1}, \cdots, t_{k}\right)$. Thus our condition, with the particular choice of $\mu(t)$ according to (3), involves a considerable simplification.

\section{REFERENCES}

1. S. Bochner, Monotone Funktionen, Stieltjessche Integrale und harmonische Analyse, Mathematische Annalen, vol. 108 (1933), p. 378.

2. - A theorem on Fourier-Stieltjes integrals, Bulletin of the American Mathematical Society, vol. 40 (1934), p. 271.

3. H. Cramér, Random Variables and Probability Distributions, Cambridge, 1937.

4. F. Hausdorf, Momentprobleme für ein endliches Intervall, Mathematische Zeitschrift, vol. 16 (1923), p. 220.

5. P. Lévy, Calcul des Probabilités, Paris, 1925.

6. I. J. Schoenberg, Remark on the preceding note by Bochner, Bulletin of the American Mathematical Society, vol. 40 (1934), p. 277.

UNIVERSITY OF STOCKHOLM,

STOCKHOLM, SWEDEN 